\begin{tabular}{|c|c|}
\hline & Volume \& Issues Obtainable at The Women University Multan \\
\end{tabular}

\title{
Cultural Impact of Perceived Parental Expectations on Students' Academic Stress
}

\author{
Muhammad Ali Talha ${ }^{1}$, Xuehong Qi $^{2}$, Muhammad Rizwan ${ }^{3}$
}

\begin{abstract}
The current study was conducted to investigate the cultural impact of perceived parental expectation on students' academic stress. Study was completed through correlational research design. Survey was conducted by using questionnaires as a method of data collection. Multistage-sampling technique was used to approach the respondents. As a sample of study 977 students were selected. There are two research instruments that were used; Perceived Parental Expectation Scale (Wang, \& Heppner, 2002) and Questionnaire of Academic Stress in Secondary Education (Garcia-Ros, Perez-Gonzalez, \& Tomas, 2018). Following statistical tests were used to analyze the data; Pearson Product Moment Correlation Coefficient, Linear regression, t-test for independent samples design, and Analysis of Variance (ANOVA). Results revealed that a positive relationship between parental expectation and academic stress, standard regression model show significant impact of parental expectations on academic stress and difference of perceived parental expectations and academic stress with respect to the gender of the students' differences was reported in result favoring male students for higher expectations and stress. Students those parents were unemployed reported to perceive higher level of parental expectations and academic stress. Significant differences due to geographical location of zones (upper Punjab, central Punjab and southern Punjab) relative to parental expectations and academic stress was also reported. Significant results were reported according to different groups of parental education. In the domain of SES, there are differences in parental expectations and academic stress among students at secondary level.
\end{abstract}

Keywords: cultural, perceive parental, academic stress, parental expectations.

\section{Introduction}

The most significant factor that plays a leading role in the growth of human capital is education. This encourages a productive and educated community. It also opens up doors for parts of society that are socially and economically disadvantaged. The key sources of the learning process by which knowledge, skills, and experiences are passed from one generation to the next generation are teaching, training, research, and growth, underlined by the educational theory and said to be the ultimate replication of the country's socio-economic development. Parents, however, are considered to have an outstanding role in influencing the growth and development of children. Furthermore, parenting activities provide an atmosphere

\footnotetext{
${ }^{1}$ Ph.D Scholar, School of Education Sciences, Nanjing Normal University, China. 31183012@ stu.njnu.edu.cn

2 Professor, School of Education Sciences, Nanjing Normal University, China. qxuehong@126.com

${ }^{3}$ Ph.D Scholar, School of Education Sciences, Nanjing Normal University, China. 31173013@ stu.njnu.edu.cn
} 
of family socialization in which parents accomplish their objectives and convey values to their children (Economic Survey of Pakistan 2015).

Parents, as the closest environment with an individual, affect the education process. The effect can have positive or negative impacts. Support, aid, and motivation from parents can help the individual to enhance the ability in academic. It is in line with the research that shows the involvement of parents' relation positively with children's education (Wilder, 2016). The hope of parents aims to make their children's lives to be better in the future. Parents' hope arises because they want a prosperous future for their children rather than the future of themselves (Rubie-davies et al., 2010). Parents' expectation helps individuals develop the ability and talent to achieve their academic targets in life (Kaplan, Liu, \& Kaplan, 2016). However, there is a negative impact on parents' hope/expectation, especially if parents have unrealistic expectations and are not following the children's ability and capacity. It is because of parents' desire to repeat life, fix the errors, or meet their lost ambition through their children (Harris, \& Goodall, 2017).

Research about parents' hope with Asians and Caucasian people or white people shows that Asian parents have a higher standard in academics, attach importance to education and consider that children's academic success is the main goal in revealing the expectation. Same, each child has a different response and perception toward their parents' expectations. The difference in this perception is caused by subjectivity and individual differences (Deb, Strodl, \& Sun, 2015).

Academic stress is psychological distress in relation to such expected frustrations linked to academic failure (Gupta \& Khan, 1987). Due to different internal and external demands, stress has become part of the academic life of students (Reddy, Menon, \& Thattil, 2018). Parents typically set unreally high expectations, leading to severe outbreaks of stress, depression and suicide by students (Hazari, 2013).

\section{Literature Review}

In general, parental expectations are defined as the beliefs and judgments of parents about the potential accomplishments of their children as expressed in course grades, how far their children will go in school, expected the highest level of education, and so on (DiBartolo \& Rendon, 2012). Several previous studies have shown that parental expectations are positively related to the high academic success of children (Danisman, 2017), and parental expectations can have a significant long-term influence on the academic achievement of children from childhood until adolescence (Froiland, Peterson, \& Davison, 2013).

Nevertheless, relatively few research focused on the effects of high parental standards outside of young people's academic performance to discuss problems such as their depressive symptoms (Supple \& Cavanaugh, 2013). Research has also shown that parental expectations are connected to the engagement and motivation of children to academic achievement, which in turn leads to increasing their academic success (Lazarides, Viljaranta, Aunola, Pesu, \& Nurmi, 2016; Long \& Pang, 2016).

Previous studies have shown that parents with high expectations are more likely to participate in their children's performance-related activities and give their children more support, including assistance in their homework and academic success, and more education opportunities to their children (Long \& Pang, 2016). A Chinese study showed that teenagers put a strong emphasis on studying and tried hard to improve their academic results. If they 
cannot meet their parental expectations, they will become guilty, helpless and useless in connection with depression (Wang, Fan, Xue, \& Zhou, 2015).

Since high parental expectations for their children's future education and careers are connected to academic success and commitment, it has been suggested that these academic and disciplinary issues could be due to low academic expectations among Swedish parents. Parental academic expectations are about how far parents think their child will go in school, while parental expectations are about how far parents want their child to go. However, two studies found that high parental expectations or aspirations were associated with lower depression and better self-concept among children (Gutierrez, 2019). For example, parental criticism rather than expectations led to negative mental health outcomes (Harris, \& Goodall, 2017); others found that high parental expectations were related to psychological distress through how they were communicated to the child. Thus, the parent-child relationship's quality should be considered when assessing the link between parental expectations and child mental health, as has been found in a previous (Ramachandiran, \& Dhanapal, 2018).

In Pakistan's culture, parents have more expectations for their male child because of the societal perspective that a male should succeed in education well enough to look after the parents and family in the future. Hence, the present study was carried out to identify the impact of culture and parental expectations and academic stress among students of secondary schools.

\section{Significance of the Study}

This study is significant, exceptionally in education, while exploring the cultural influence on parental expectations toward their children's achievement. Asian culture is quite different from western culture, so the study's findings are contradictory in perspective Pakistan. Results of the current study is the way to know about how culture affects parental academic expectation. In the educational sector, the study will get precious importance in Pakistan and Asian countries.

\section{Objectives of the Study}

1. To explore the relationship between perceived parental expectation and academic stress among students of medical classes

2. To investigate the moderating effect of the culture between the relationship of parental expectation and students' academic stress

3. To compare the differences in parental expectation and academic stress between male and female students

4. To determine the differences in demographic variables (father's occupation, mother's occupation, father's education, mother's education)

5. To identify the role of demographic characteristics in perceiving parental expectations for academic stress among students of medical classes

\section{Research Questions}

1. What is the relationship between perceived parental expectation and academic stress?

2. What is moderating effect of culture between the relationship of perceived parental expectation and students' academic stress?

3. What is the difference between perceived parental expectation and academic stress? 
4. What is the level of perceived parental expectation and academic stress in the perspective of demographic variables?

5. What is the role of demographic variables in perceiving parental expectation?

\section{Methodology}

\section{Research Design}

The present study was completed through a correlational research design in the domain of the positivist paradigm. The demographic information of the participants was comprised of the following characteristics; gender (males and females), zone (North Punjab, Central Punjab, and South Punjab), parents' education (uneducated, primary, matric, bachelor, master or above), parents' occupation (unemployed and employed).

\section{Participants}

The population of the study was comprised of the students of higher secondary schools in Punjab province, Pakistan. The current study's target population was divided into three zones of Punjab province (north Punjab, central Punjab, and south Punjab). The survey was used as a method of data collection from the respondents. A multistage sampling technique was used to approach the participants. As a sample of the study, 977 students were selected; among them, 524 were males, and 453 were females.

\section{Instruments}

1. Perceived Parental Expectation

2. Questionnaire of Academic Stress in Secondary Education

\section{Perceived Parental Expectation}

The perceived Parental Expectation scale was developed by (Wang, \& Heppner, 2002). The first factor, consisting of 16 items, was titled "Personal Maturity (PM)." "Parents expect me to control/change my bad temper," "Parents expect me not to offend them verbally/vocally," and "Parents expect me to behave maturely." This factor was defined as expectations related to how appropriate an individual's manners were in the perspective of Pakistan's culture, such as exercising self-control, politeness, responsibility, maturity, and obedience. The second factor, labeled "Academic Achievement (AA)," was composed of 9 items. "Parents expect my academic performance to make them proud," "Parents expect me to have excellent academic performance," and "Parents expect me to perform better than others academically." This factor was defined as expectations related to an individual's success in pursuing a career and academic performance. The third factor, titled "Dating Concerns (DC)," consisted of 7 items. "Parents expect me to find someone who has a good financial status when dating," "Parents expect me to find someone with an advanced degree when dating," and "Parents expect me to select my dates with his/her family background in mind." This factor was defined as expectations related to one's dates and potential significant other or marital partner. (PPE; $1=$ not at all expected, $6=$ very strongly expected).

\section{Questionnaire of Academic Stress in Secondary Education}

Questionnaire of Academic Stress in Secondary Education was developed by (GarciaRos, Perez-Gonzalez, \& Tomas, 2018). Thus, the initial version of the QASSE used in the 
study comprised 30 items related to different potentially stress-producing secondary education situations. Students' responses reveal their stress levels in the different school situations on a Likert-type scale with five response options $(1=$ "Very low," to $5=$ "Very high"). The 30-item draft was submitted and administered to six secondary school teachers and 18 secondary students (12 from compulsory secondary education and six from postcompulsory secondary education), using a Likert-type response scale from 1 (very low) to 5 (very high). Feedback obtained from the teachers and students emphasized the relevance and applicability of all the items, and minor adjustments were made in the wording of various items to make the vocabulary more easily understood by adolescents.

\section{Procedures}

Informed consent was taken after receiving permission for the data collection from the higher secondary schools' administration. All the questions were arranged into a booklet according to perceived parental expectation and students' academic stress. A booklet was given to each student to take the response by reading the instructions provided on the research instrument. It was assured to students that their information would be kept confidential and only used for the study.

\section{Data Analysis}

The results were analyzed quantitatively from the statistical package for social sciences (SPSS). Descriptive and inferential statistics were used. Descriptive as well as inferential statistics were used to analyze the data. Statistical Package of Social Sciences (SPSS) was used for data analysis. Following statistical tests were used to test hypotheses; Pearson Product Moment Correlation Coefficient, Linear regression, t-test for independent sample design, and Analysis of Variables (ANOVA).

\section{Results}

\section{Descriptive Statistics}

Table 1: Demographic Variables

\begin{tabular}{|l|l|l|l|}
\hline Name & Frequency & Percent & $\begin{array}{l}\text { Cumulative } \\
\text { Percentage }\end{array}$ \\
\hline 1. Gender & & & \\
\hline Male & 524 & 53.6 & 53.6 \\
\hline Female & 453 & 46.4 & 100.0 \\
\hline 2. Zone & & & \\
\hline North Punjab & 253 & 25.9 & 25.9 \\
\hline Central Punjab & 371 & 38.0 & 63.9 \\
\hline Southern Punjab & 353 & 36.1 & 100.0 \\
\hline 3. Parental Education (PE) & & & \\
\hline Uneducated & 319 & 32.7 & 32.7 \\
\hline Primary & 318 & 32.5 & 65.2 \\
\hline Metric & 134 & 13.7 & 78.9 \\
\hline Bachelor & 51 & 5.3 & 84.1 \\
\hline Master and above & 155 & 15.9 & 100.0 \\
\hline 4. Occupation & & & \\
\hline Unemployed & 479 & 49.0 & 49.0 \\
\hline
\end{tabular}




\begin{tabular}{|l|l|l|l|}
\hline Employed & 498 & 51.0 & 100.0 \\
\hline $\begin{array}{l}\text { 5. Socioeconomic Status } \\
\text { (SES) }\end{array}$ & & & \\
\hline Low & 478 & 48.9 & 48.9 \\
\hline Middle & 202 & 20.7 & 69.6 \\
\hline High & 297 & 30.4 & 100.0 \\
\hline
\end{tabular}

The participants' demographic information is described in this table; 524 male and 453 female respondents were selected from three regions of the Punjab province; Northern Punjab, Central Punjab, and Southern Punjab. The number of respondents is 253, 371, and 353 from the north, central Punjab regions of Punjab. The parental education is 319,318 , 134, 51, and 155 in relation to uneducated, primary metric bachelor and master/ above, respectively. The students' parental occupation is categorized as unemployed (479) and employed are (498). Socio-economic status is composed of low (487), middle (202), and high (297) categories.

\section{Content Analysis: Analytical Method}

There are the following statistical method which was used to analyze the data

1. Cronbach's Alpha Reliability

2. Pearson Product- Movement Correlation

3. Linear Regression

4. T-test for Independent Sample Designs

5. Analysis of Variance

Table 2: Cronbach's Alpha Reliability

\begin{tabular}{|c|c|c|}
\hline Variables & Number of Items & Cronbach's Alpha \\
\hline Parental Expectation & 32 & .991 \\
\hline Academic Stress & 30 & .977 \\
\hline
\end{tabular}

The instruments' reliability was checked through Cronbach's Alpha Reliability, which indicates that all instruments in the perspective of Pakistan's culture. The values of Cronbach's Alpha are significant. So, these research instruments are considered reliable to measure the perception of parenting expectations and academic stress. The Cronbach's Alpha value of parenting expectations (.991) and academic achievement (.977).All these figures represent that scales are reliable and significant.

Table 3: Correlation Matrix among Perceive Parenting Expectations and Students Academic Stress

\begin{tabular}{|c|c|c|c|c|c|}
\hline & & Mean & Std. Deviation & 1 & 2 \\
\hline 1 & PPE & 123.8864 & 41.99530 & 1 & $.942^{* *}$ \\
\hline 2 & AS & 29.02040 & 29.02041 & & 1 \\
\hline
\end{tabular}

Note,

PPE Perceive parental expectations

AS Academic stress

Table 3 shows the relationship between parental expectation and Academic stress among students at the secondary level of education Findings. The study reveals a positive 
relationship between parental expectation and academic stress as it was hypothesized that there would be a positive correlation between parental expectation and academic stress. Results support the hypothesis. Furthermore, the relationship between variables is positive. Findings of the study revealed that in Pakistan culture, parental expectations are high compared to other cultures concerning academic accomplishment; therefore, results are highly correlated with students' perception of parental expectations as enhancing academic stress.

Table 4: Standard Regression Model showing impact of Perceive Parental Expectations on Academic stress

\begin{tabular}{|c|c|c|c|c|c|}
\hline Predictor & B & Std. Error & Beta & t-test & P-value \\
\hline (Constant) & 22.763 & 1.705 & & 23.511 & .000 \\
\hline PPE & .651 & .007 & .942 & 87.983 & .000 \\
\hline
\end{tabular}

$\mathrm{R} 2=.888$, Adjusted R2 $=.888,(\mathrm{~F}(1,976)=7740.976, \mathrm{p}<0.05)$

Table 4 describes the impact of perceiving parenting expectations on students' academic achievement. The study results indicate that a positive impact of parental expectation was reported on the students' academic stress. Therefore, Perceive Parenting expectations predict students' academic stress positively, as parents in Asian culture are more concerned about their children's academic accomplishment. Findings reveal that impact is significant, demonstrating that parental expectation predicts $88 \%$ of academic stress among students.

Table 5: Mean, Standard Deviation, t-value and scores of Perceived Parental expectation and academic stress with respect to the gender of students $(n=977)$.

\begin{tabular}{|c|c|c|c|c|c|c|c|}
\hline Variable & Gender & $\mathrm{N}$ & $\mathrm{M}$ & Std. Deviation & $\mathrm{df}$ & t-test & $\mathrm{p}$-value \\
\hline \multirow{2}{*}{ PPE } & Male & 524 & 138.2424 & 46.43304 & 975 & \multirow{2}{*}{21.352} & \multirow{2}{*}{.000} \\
& Female & 453 & 107.2804 & 28.25153 & & & \\
\multirow{2}{*}{ As } & Male & 524 & 109.6355 & 31.72430 & 975 & \multirow{2}{*}{7.366} & \multirow{2}{*}{.000} \\
& Female & 453 & 96.2804 & 23.62471 & & \\
\hline
\end{tabular}

This table shows the difference in perceived parental expectations and academic stress concerning the gender of the students. According to the researcher s' hypothesis, the difference will be significant at the secondary level. Results revealed that from the perspective of Punjab culture. Male students reported that their parents have more expectations toward their academic achievements; therefore, due to higher parental expectations, male students feel more parental expectations and greater academic stress than their female counterparts. Collectively, the difference was founded as significant between male students and female students due to parental expectation.

Table 6: Mean, Standard Deviation, t-value and scores of Perceived Parental Expectations on Academic Stress with respect to the parental occupation $(n=977)$

\begin{tabular}{|c|c|c|c|c|c|c|c|}
\hline Variable & $\begin{array}{c}\text { Parental } \\
\text { Occupation }\end{array}$ & $\mathrm{N}$ & $\mathrm{M}$ & $\begin{array}{c}\text { Std. } \\
\text { Deviation }\end{array}$ & $\mathrm{df}$ & t-test & $\mathrm{p}$-value \\
\hline \multirow{2}{*}{ PPE } & Unemployed & 479 & 130.6305 & 2.02265 & 975 & 4.983 & .000 \\
\hline \multirow{2}{*}{$\mathrm{AS}$} & Employed & 498 & 117.3996 & 1.73125 & & \\
& Unemployed & 479 & 29.20244 & 29.20244 & 975 & 5.050 & .000 \\
\hline
\end{tabular}


This table determines the comparison of perceived parenting expectations on students' academic stress in respect to the parental occupation (employed and unemployed). Students whose parents were unemployed reported perceiving a higher level of parental expectations. Results reveal a significant comparison according to parental expectation as demographic characteristics similarly; academic stress is higher among unemployed parents. Therefore, they have greater academic stress. The findings of the study showed a significant difference in research variables in terms of parental occupation. Academic stress was reported more among unemployed families' children than employed ones because parents view the education system to earn a handsome amount of salary and means of earning. Consequently, the differences between employed and unemployed parents' expectations occur that influence students' academic stress.

Table 7: Analysis of variance (one-way) showing the differences of Perceived Parental expectation and academic stress in relation to zone $(n=977)$.

\begin{tabular}{|c|l|l|l|l|l|}
\hline & Sum of Squares & df & Mean Square & F & p-value \\
\hline PPE Between Groups & 168253.138 & 2 & 84126.569 & 52.761 & .000 \\
Within Groups & 1553025.251 & 974 & 1594.482 & & \\
$\quad$ Total & 1721278.389 & 976 & & & \\
\hline AS Between Groups & 81927.502 & 2 & 40963.751 & 53.914 & 000 \\
Within Groups & 740043.5961 & 974 & 759.798 & & \\
Total & 821971.097 & 976 & & & \\
\hline
\end{tabular}

This table shows the multiple comparisons of perceived parental expectations and academic stress about Zone (upper, central, and southern part) among students at the secondary level. It was hypothesized that differences would be significant. In the perspectives of parental expectation and academic stress, a significant difference is reported. Findings of the study supported significant differences due to the geographical location of zones, which means upper Punjab has a different level of expectation and academic stress compared to central Punjab and southern Punjab level of parental expectations academic stress.

Table 8: Analysis of variance (one way) showing the differences of Perceived Parental expectation and academic stress in relation to Parental education level $(n=977)$

\begin{tabular}{|c|l|l|l|l|l|}
\hline & Sum of Squares & df & Mean Square & F & p-value \\
\hline PPE Between Groups & 224340.111 & 4 & 56085.028 & 36.417 & .000 \\
Within Groups & 1496938.278 & 972 & 1540.060 & & \\
Total & 1721278.389 & 976 & & & \\
\hline AS Between Groups & 125545.784 & 4 & 31386.446 & 43.806 & 000 \\
Within Groups & 696425.313 & 972 & 716.487 & & \\
Total & 821971.097 & 976 & & & \\
\hline
\end{tabular}

This table describes the mean difference between parenting expectation and academic stress concerning parental education level. Results of the study indicated that differences are significant according to different groups of parental education. The results show that parental education level causes differences in parental expectations of their children, which causes academic stress among students. Findings reveal that parental education levels are different from each other about expectations from children and perception of academic stress among students. 
Table 9: Analysis of variance (one way) showing the differences of Perceived Parental expectation and academic stress in relation to parental socio economic status $(n=977)$.

\begin{tabular}{|c|l|l|l|l|l|}
\hline & Sum of Squares & df & Mean Square & F & p-value \\
\hline PPE Between Groups & 131421.845 & 2 & 65710.923 & 40.257 & .000 \\
Within Groups & 1589856.544 & 974 & 1632.296 & & \\
$\quad$ Total & 17121278.389 & 976 & & & \\
\hline AS Between Groups & 113495.780 & 2 & 56747.890 & 78.016 & .000 \\
Within Groups & 708475.318 & 974 & 727.387 & & \\
Total & 821971.097 & 976 & & & \\
\hline
\end{tabular}

This table explores the difference in parental expectations about parental socioeconomic status. In the SES domain, there are differences in parental expectations and academic stress among students at the secondary level. According to results, differences demonstrate that parental expectations and academic stress are different on different socioeconomic status levels. So, parental socioeconomic status differentiates the degree of parental expectation and academic stress.

\section{Discussion}

Parenting is a keyword, which includes all forms of styles parents adopt while dealing with their children. These styles influence the way a child grows up and perceive things. Good parenting can lead to a responsible citizen. The attitude and behavior that an apparent show towards a child has a significant impact on the child's behavior and attitude in general life. Parents and children must have a positive and healthy relationship if they are to become confident, independent, and have the necessary social skills. The parenting styles influence these relations between a parent and a child. The parenting styles adopted by people are defined as the attitudes, behaviors, values, morals, mind-set, and principles that a parent shows towards a child and these behavior attitudes are expected to be adopted by the child.

Undoubtedly, culture has a great impact on the kind of parenting styles adopted by parents. Cultural norms dictate what type of parenting style would be adopted, what values and morals should be given to the child, and what acceptable behaviors. The Asian culture, for instance, is based on submission towards parents. Parents are placed at the top of the ladder. A lot of focus is put on the way a child is raised. The Asian culture emphasizes societal status, which can be ruined if anyone from the family does something out of the norms. This makes it a great concern for parents, and they want to control every aspect of their child's life so that he/she would not do anything to taint the family name.

The first table of descriptive statistics describes the participants' demographic information as 524 male and 453 female respondents selected from three regions of Punjab from Northern Punjab, Central Punjab, and Southern Punjab. The total number of respondents is 977 . The category of parental education has five groups (uneducated, primary, metric, Bachelor, master, and above). Forth demographic variable is parents' occupation as divided into two groups employed and unemployed. Next is socioeconomic status (low, middle, and high).

Cronbach's Alpha Reliability, Pearson Product- Movement Correlation, Linear Regression-test for Independent Sample Designs, and Analysis of Variance are used to inferences about the data. Cronbach's Alpha Reliability, which indicates that all instruments in the perspective of Pakistan's culture. The values of Cronbach's Alpha are significant. So, 
these research instruments are considered reliable to measure the perception of parenting expectations and academic stress.

Table 3 shows the relationship between parental expectation and Academic stress among students at the secondary level of education. The findings of the study show a positive relationship between parental expectation and academic stress. When parental expectations increase, the students perceive more academic stress as they want to fill full their parent's expectations and thought if they were unable to meet the standards of parental expectations, their parents would be disappointed; therefore, their perception of academic accomplishment exceeds and fear of failure leads them to academic stress. Findings of the study revealed that in Pakistan culture, parental expectations are high compared to other cultures about the academic accomplishment; therefore, results are highly correlated with students' perception of parental expectations and academic stress.

Table 4 describes the impact of perceiving parenting expectations on students' academic achievement. Outcomes highlight the significant impact of parental expectations and academic stress up to $88 \%$. When parents have high expectations from their children, they put more pressure on their children to fulfill those expectations.

Nobody wants to be disappointed, and parents are the same. When they have high expectations, they want those expectations to be fulfilled. That is why they put pressure on their child to meet those expectations. If parents do not have high expectations from their children, they do not put pressure on them.

Table 5 shows that the gender of students has an impact on academic stress and parental expectations. Collectively, the difference was found significant between male students and female students due to parental expectations as parents expect more good grades from their male child. From the beginning, males were considered to be the breadwinner of the family, while females were thought to be as someone who will, one day, leave the house. That is why; more focus is put on boys to do well in academics as a good academic grader will mean a better job, which means a better life. That is why males are pressured more to do well in academics.

Table 6 determines the mean comparison of perceived parenting expectations and academic stress regarding the parental occupation (employed and unemployed). Students whose parents were unemployed reported perceiving a higher level of parental expectations and academic stress. This is because it is very hard to live a financially stable life in today's economy. The economy of today's world is managed, making it harder for people with lowpaying jobs or no jobs to live peacefully. Thus, unemployed parents put more pressure on their child to do well academically so that their child can get a good job and take the family's financial responsibility since they cannot take the burden themselves. Table 7 shows the multiple comparisons of perceived parental expectations and academic stress about Zone (upper, central, and southern) students. The study's findings supported that there are significant differences among upper Punjab compared to central Punjab and southern Punjab level of parental expectations and academic stress. The reason might be that upper Punjab is a cosmopolitan area. People whose families are financially well off are the ones who usually live there. Thus, they do not have to pressurize their child to get good grades. Central Punjab has a much higher academic stress because the lifestyle there requires you to have a highpaying job, which is very hard without a proper education level. In the lower Punjab, the living standards are not that high, but still, parents do pressurize their children to some extent to get good grades so they can also live a better life. 
Table 8 indicated that differences are significant according to different groups of parental education. The results show that parental education level causes differences in parental expectations of their children, which causes academic stress among students. Findings reveal that parental education levels are different from each other about expectations from children's and perception of academic stress among students. Uneducated parents usually do not pressure their children to get good grades because they do not understand the value of education. Parents who are well educated, on the other hand, have high expectations from their kids to do well in academics because they understand the importance of academic achievements. Parents who are a little educated also are optimistic about their child because they also understand the importance of education. Table 9 explores the difference of parental expectations about parental socioeconomic status, showing differences are significant, demonstrating that parental expectations and academic stress are different on a different level of socioeconomic status. Parents belonging to a high-status family do not have high hopes or expectations from their children because they don't need to. They are well off enough even if their child doesn't get a good job. They are financially stable. Parents from the middle class or lower class, on the other hand, have high expectations because if their child cannot get a good job, then they will have to deal with a serious financial crisis.

\section{Conclusion}

The impact of culture cannot be neglected in practicing parenting styles. Cultural norms dictate parenting style, and values and morals should be given to the child and what behaviors are acceptable or non-acceptable. Results show a significant positive relationship between parental expectation and academic stress; standard regression model shows a significant impact of parental expectations on academic stress and difference of perceived parental expectations and academic stress concerning the gender of the students' differences was reported in result favoring male students for higher expectations and stress. Students whose parents were unemployed reported perceiving a higher level of parental expectations and academic stress. Significant differences due to zones' geographical location (upper Punjab, central Punjab, and southern Punjab) relative to parental expectations and academic stress were also reported. Significant results were reported according to different groups of parental education. In the SES domain, there are differences in parental expectations and academic stress among students at the secondary level.

There are many factors for these expectations. Societal standards, cultural norms, financial status all come into play. Higher pressure is placed on boys than girls as boys are culturally considered the breadwinner of the family. High expectations are associated with children of middle-class families, as they need to secure a financially stable future, which looks most promising through a good degree. On the other hand, Parents from high-status families do not pressure of their children to do well as they are already financially strong. Similarly, educated people put more pressure on their children as they understand the importance and need of a good education. However, higher expectations than the child's aptitude have destructive effects on the child's mental health. It can ruin the child's mental and physical health, especially if the child never gets emotional support from his/her parents.

\section{Practical Implication}

1. This study has a geographically widespread sample that contributes to the better generalization of results.

2. This study helps to recognize Pakistan's culture, parenting styles, ethics, and social influences on students' perception and academic stress. 
3. The research has both theoretical and practical applications. To increase children's academic achievement, the study focuses on parenting styles as these factors are of great significance for practical implication.

4. It highlights common parenting styles practice in Pakistan.

5. It helps understand the parents' psychology and a more in-depth view of parenting styles and practice is obtained.

6. This study helps to provide the best possible aid to deal with academic stress.

7. Use of mixed methods enhanced the quality of this research project which has provided exciting results that would be useful for parents who involve in the mathematics education of their children

\section{Limitations of the Research}

1. The study was conducted in a period of a pandemic lockdown (COVID-19), and the effects of such variables on the academics can alter the results from those in conditions.

2. Stress, depression, and anxiety among students were not considered when studying parenting styles and academic achievements for this study.

3. The evolution of parenting styles' impact on academic achievement was not carried with a longitudinal design.

4. Period was too small for conducting this study and to analyze the data effectively and efficiently.

5. There is restrain of money and resources for this study.

\section{Recommendations for Future Research}

1. In the Future, the research should be carried out on larger scales.

2. Psychiatric disorders, incredibly stress, depression, and anxiety among students, should be considered when studying parenting styles and academic achievements.

3. Theoretically, the best environment for a child would be one in which there are clear communications of expectations on the side of the parent and admittance of perceived weaknesses on both parent's and child's part. Future researches should test this theory.

4. Future researches should study the impact of time in the academic environment on the variables.

5. Impact of changes in educational policies and curriculum should also be studied in detail.

6. The change in parenting styles should be studied. We need to consider the increase in stress during proximity of examinations and its effects on a child's academic performance.

\section{References}

Danişman, Ş. (2017). The effect of expectation on student achievement. In The Factors Effecting Student Achievement (pp. 227-245). Springer, Cham.

Deb, S., Strodl, E., \& Sun, J. (2015). Academic stress, parental pressure, anxiety and mental health among Indian high school students. International Journal of Psychology and Behavioral Sciences, 5(1), 26-34. 
DiBartolo, P. M., \& Rendón, M. J. (2012). A critical examination of the construct of perfectionism and its relationship to mental health in Asian and African Americans using a cross-cultural framework. Clinical Psychology Review, 32(3), 139-152.

Froiland, J. M., Peterson, A., \& Davison, M. L. (2013). The long-term effects of early parent involvement and parent expectation in the USA. School Psychology International, 34(1), 33-50.

Gutierrez, B. D. (2019). First-Generation College Graduates' Perceptions of Their Parental Expectations (Doctoral dissertation, St. Thomas University).

Harris, A., \& Goodall, J. (2017). Engaging Parents in Raising Achievement-Do They Know They Matter. Univ Warwick, 1-93.

Hazari, A. (2013). The biggest stress for students: parental pressure.

Kaplan, D. S., Liu, X., \& Kaplan, H. B. (2016). Influence of parents' self-feelings and expectations on children's academic performance. The Journal of Educational Research, 94(6), 360-370.

Lazarides, R., Viljaranta, J., Aunola, K., Pesu, L., \& Nurmi, J. E. (2016). The role of parental expectations and students' motivational profiles for educational aspirations. Learning and Individual Differences, 51, 29-36.

Long, H., \& Pang, W. (2016). Family socioeconomic status, parental expectations, and adolescents' academic achievements: A case of China. Educational Research and Evaluation, 22(5-6), 283-304.

Ramachandiran, M., \& Dhanapal, S. (2018). Academic Stress among University Students: A Quantitative Study of Generation Y and Z's Perception. Pertanika Journal of Social Sciences \& Humanities, 26(3).

Ramli, N. H., Alavi, M., Mehrinezhad, S. A., \& Ahmadi, A. (2018). Academic stress and self-regulation among university students in Malaysia: Mediator role of mindfulness. Behavioral Sciences, 8(1), 12.

Reddy, K. J., Menon, K. R., \& Thattil, A. (2018). Academic stress and its sources among University students. Biomedical and Pharmacology Journal, 11(1), 531-537.

Rubie-Davies, C. M., Peterson, E., Irving, E., Widdowson, D., \& Dixon, R. (2010). Expectations of achievement: Student teacher and parent perceptions. Research in Education, 83(1), 36-53.

Supple, A. J., \& Cavanaugh, A. M. (2013). Tiger mothering and Hmong American parentadolescent relationships. Asian American Journal of Psychology, 4(1), 41.

Wang, M., Fan, C., Xue, N., \& Zhou, Z. (2015). Home stressors influence adolescents' depressive symptoms: Rumination as a mediator. Journal of Psychological Science, 38(6), 1398-1403.

Wilder, S. (2014). Effects of parental involvement on academic achievement: A metasynthesis. Educational Review, 66(3), 377-397. 